\title{
Time to implement 9-month infant HIV testing in South Africa
}

\author{
L Fairlie, ${ }^{1} \mathrm{MB}$ ChB, FCPaed (SA), MMed; C A Madevu-Matson, ${ }^{1,2}$ MSc; V Black, ${ }^{1} \mathrm{MD}, \mathrm{MSc}$; G G Sherman, ${ }^{3} \mathrm{MB}$ BCh, PhD \\ ${ }^{1}$ Wits Reproductive Health and HIV Institute, University of the Witwatersrand, Johannesburg, South Africa \\ ${ }^{2}$ ICAP at Columbia University, Mailman School of Public Health, Columbia University, New York, USA \\ ${ }^{3}$ Department of Paediatrics and Child Health, Faculty of Health Sciences, University of the Witwatersrand, Johannesburg, \\ South Africa, and Centre for HIV and STI, National Institute for Communicable Diseases, Johannesburg
}

Corresponding author: L Fairlie (lfairlie@wrhi.ac.za)

Background. South Africa (SA) is likely to meet the National Strategic Plan target of $<2 \%$ mother-to-child HIV transmission at 6 weeks of age in 2015. Children infected with HIV after 6 weeks often remain undiagnosed because of poor implementation of post-weaning and final outcome 18-month HIV testing. The World Health Organization recommends a screening HIV rapid test (HRT) in HIV-exposed infants at the 9-month immunisation visit to exclude postnatal infection, with a confirmatory HIV polymerase chain reaction (PCR) test if the HRT is positive.

Objective. To evaluate the impact of substituting this recommendation for the post-weaning HIV testing recommended by SA guidelines. Methods. Rates of seroreversion and probability of infection at 9 months of age were applied to a theoretical population of $100 \mathrm{HIV}$-exposed infants, uninfected at birth and breastfed for 1 year with antiretroviral prophylaxis. Nine scenarios were developed and the number of HIV PCRs saved compared with current guidelines was calculated.

Results. Nine-month testing using the HRT reduced the number of follow-up PCR tests done in all scenarios by $>50 \%$, with differences ranging from $51 \%$ to $59 \%$ and $81 \%$ to $89 \%$ for low and high seroreversion rates, respectively.

Conclusions. Nine-month testing using HRT would increase identification and early treatment of HIV-infected infants, improve monitoring of postnatal transmission rates, and reduce the number of HIV PCR tests done with resultant cost saving. Training of healthcare workers implementing HRT would be required. Ongoing efforts to improve implementation and monitoring of testing at 9 and 18 months will be essential.

S Afr Med J 2015;105(9):765-768. DOI:10.7196/SAMJnew.8175

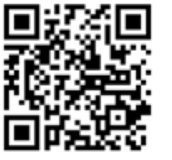

In South Africa (SA), excellent gains have been made since 2002 in prevention of mother-to-child transmission (PMTCT) of HIV, with $>90 \%$ of HIVinfected women accessing PMTCT. ${ }^{[1]}$ The national early transmission rate of HIV infection at around 6 weeks of age is measured by the District Health Information System, the National Health Laboratory Service and the 2010 - 2013 South African PMTCT Evaluation (SAPMTCTE) studies and is on track to meet the National Strategic Plan target of $<2 \%$ in 2015..$^{[2-4]}$ To detect postnatal transmission of HIV, national guidelines recommend that HIV-exposed uninfected (HEU) infants undergo repeat HIV testing 6 weeks after weaning and at 18 months of age. With dwindling early vertical transmission rates as a result of SA's successful PMTCT programme, proportionately more infants and children diagnosed after 6 weeks of age will contribute to the paediatric HIV epidemic, and it is vital that they are identified.

HIV testing after weaning and at 18 months is poorly implemented and monitored, with post-weaning HIV testing being particularly problematic because in practice it lacks a specific time point. Exclusive breastfeeding for the first 6 months of life under antiretroviral therapy (ART) cover for HIV-exposed infants has been recommended in SA since 2010, with continued breastfeeding to 12 months of age ${ }^{[5]}$ After the 6-week HIV polymerase chain reaction (PCR) test, no HIV test is scheduled for a period of 1 year for the majority of breastfed HEU infants at daily risk of postnatal transmission, unless they are weaned earlier or become symptomatic. Coverage of 18 -month testing for HEU infants is also poor and data from programmatic settings are scarce. Grimwood et al. ${ }^{[6]}$ of Kheth'Impilo report HIV positivity rates in children tested at 18 months of $10.7 \%$ in 2009 , reduced to $3.8 \%$ in 2011; however, only $24 \%$ of HIV-exposed children were tested. The rate of postnatal transmission and the extent of the paediatric HIV epidemic in SA therefore remain unmeasured and HIV-infected infants and children remain unidentified.

Factors that increase the risk of postnatal transmission are maternal infections not diagnosed during pregnancy, infection acquired late in pregnancy or during breastfeeding, poor adherence to ART during breastfeeding, and mixed infant feeding practices. ${ }^{[7,8]}$ Exclusive breastfeeding rates are low, with the SAPMTCTE reporting rates of $35.5 \%$ at 6 weeks in $2010{ }^{[4]}$ Furthermore, there is mounting evidence that the 6-week HIV PCR test yields false-negative results in the presence of maternal and/or infant antiretroviral prophylaxis. ${ }^{[9]}$ These infants are likely to be diagnosed later in life, with their infection attributed to postnatal transmission.

A new routinely offered and provider-initiated testing strategy is required in SA to ensure testing of infants exposed to HIV through breastmilk and those who are missed in the first few months of life. Since 2008, World Health Organization (WHO) guidelines for infant HIV diagnosis have recommended using an antibody detection assay such as an HIV rapid test (HRT) at 9 months of age for all HEU infants, with a confirmatory HIV PCR test if the HRT is positive. ${ }^{[10]}$ HRTs performed on whole blood are able to detect seroreversion (the loss of maternal HIV antibodies) in the majority of HEU infants by $8-10$ months of age, depending on the HRT used. ${ }^{[11]}$ This means that a carefully selected HRT routinely offered at the 9-month Expanded Programme on Immunization (EPI) visit could exclude HIV infection in $80-100 \%$ of HEU infants. Measles 
coverage rates at 9 months (MCV1) in SA in 2010 were reported to be $95 \%$, higher than the $83 \%$ in the same year of the second measles vaccine (MCV2), suggesting that if 9-month HRT were introduced at the 9-month measles vaccine visit, coverage of HIV-exposed infants might potentially be greater than at the 18 -month visit. ${ }^{[12]}$ The vast burden of testing costs in an infant HIV testing programme lies in the number of HIV PCR tests performed, costing between ten and 30 times more than any HRT used in SA (Prof. Gayle Sherman, personal communication). An inexpensive screening test that coincides with a routine (EPI) visit at 9 months will diagnose HIV-infected infants early at a routine visit, provide data to monitor transmission at 9 months and plan for the numbers of children requiring combination antiretroviral therapy (cART), facilitate initiation of cART to prevent morbidity and mortality, and assist with identifying gaps in prevention programmes. Additionally, performing HRT will reduce the costs associated with the more expensive HIV PCR test, which will be unnecessary in infants testing HIV-negative on HRT.

\section{Objective}

To evaluate the impact of introducing a screening HRT test for HEU infants at the routine 9-month EPI visit for measles immunisation in line with WHO recommendations, with a confirmatory HIV PCR test for infants testing positive on HRT, instead of a post-weaning HIV PCR test.

\section{Methods}

Estimating the number of HIV PCR tests avoided with 9-month HRT

Under current National Department of Health $(\mathrm{NDoH})$ guidelines, all infants who ever start breastfeeding require one HIV PCR test after cessation of breastfeeding (assuming they are $<18$ months of age), occurring around 13.5 months of age in those breastfed until 12 months. In the testing proposed in this article, all infants who ever start breastfeeding will be tested with a screening HRT at 9 months and a confirmatory HIV PCR test if the HRT is positive. Only infants who test positive on the HRT at 9 months, which would include infants who are HIV-infected and those who have not yet seroreverted, therefore require an HIV PCR test. If an infant is HIV-uninfected but still breastfeeding at the 9-month visit, they would receive a followup HRT at 18 months as per the current guidelines unless they became symptomatic (Fig. 1).
To directly compare the number of HIV PCR tests performed under current guidelines with the proposed 9-month HRT testing, a model was constructed for a population of 100 breastfeeding HEU infants and restricted to include only the HIV PCR tests performed after weaning and after a positive HRT. Breastfeeding exposure was assumed to be $100 \%$ and to continue to 12 months of age, as recommended in the $\mathrm{WHO}$ and $\mathrm{NDoH}$ guidelines. The model did not include recently introduced targeted birth and 16-week testing, the 6-week HIV PCR, clinically indicated testing in symptomatic children, and testing at or after 18 months of age, because the numbers of these tests would not differ between the $\mathrm{NDoH}$ guidelines and the proposed testing.

Nine scenarios were developed that factored in a combination of low, middle and high levels of both seroreversion and the probability of becoming HIV-infected over 9 months of breastfeeding. ${ }^{[8,11,13]}$ Ranges

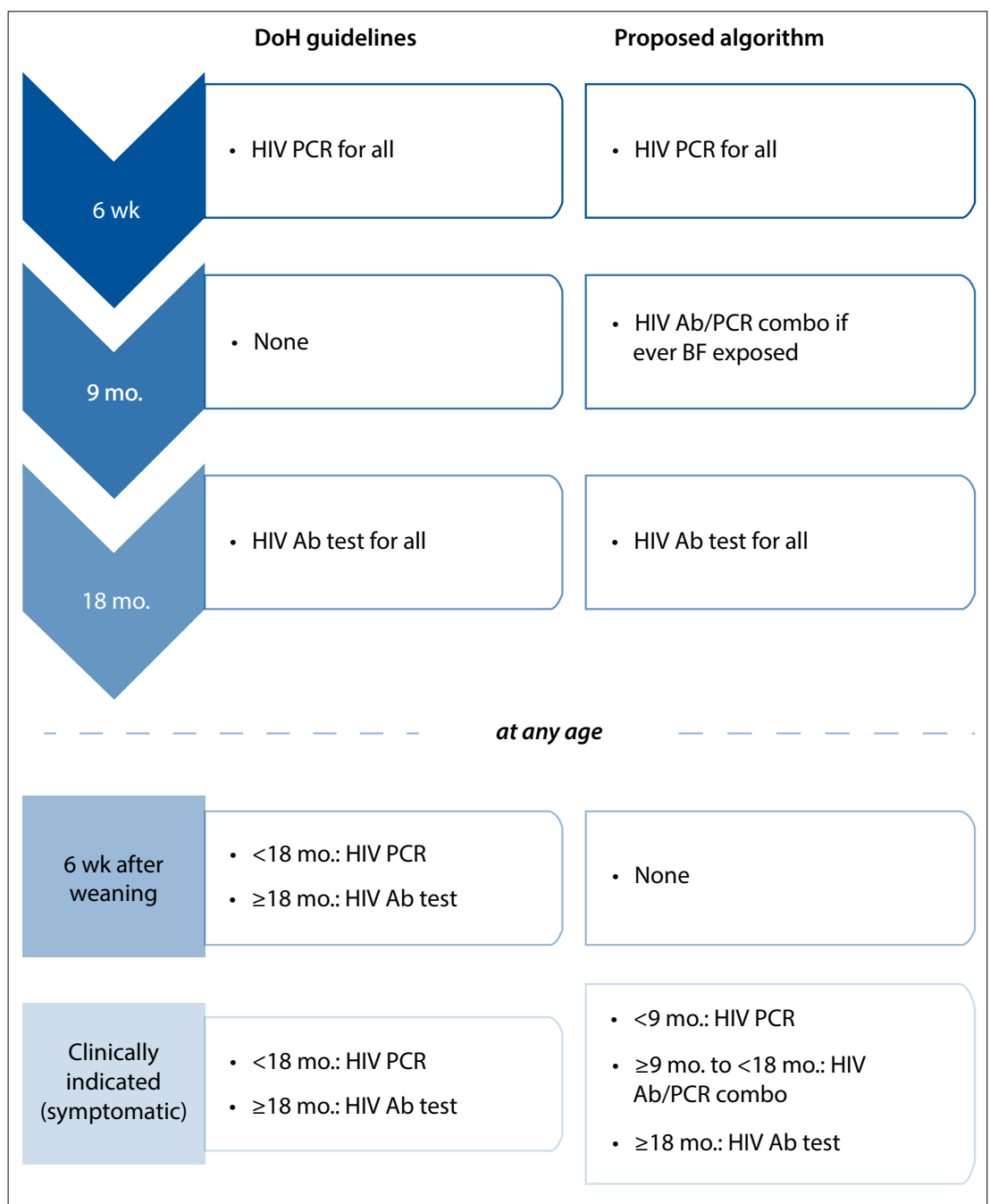

Fig. 1. Guideline for service delivery models for exposed infant HIV testing. HIV testing by age and timing for all HEU infants included in current $\mathrm{DoH}$ guidelines and proposed 9-month HRT algorithm. (BF= breastfed; $A b=$ antibody; $A b / P C R$ combo $=A b$ test screening, confirmatory $P C R$ if $A b$ test is positive.) 


\begin{tabular}{|c|c|c|c|}
\hline & \multicolumn{3}{|c|}{ Seroreversion at 9 months } \\
\hline & Low $(60 \%)$ & Mid (80\%) & High (90\%) \\
\hline \multicolumn{4}{|c|}{$\begin{array}{l}\text { Probability of } \\
\text { infection per month of } \\
\text { breastfeeding }\end{array}$} \\
\hline Low $(0.0016)$ & 59 & 79 & 89 \\
\hline Mid (0.0020) & 58 & 78 & 88 \\
\hline High (0.0100) & 51 & 71 & 81 \\
\hline \multicolumn{4}{|c|}{$\begin{array}{l}\text { *Using ranges described in published literature for HIV exposed infant seroreversion rates } \\
\text { at } 9 \text { months of age }(\sim 80 \% \text {, range } 60-90 \%) \text { and the probability of infant HIV infection per } \\
\text { month of breastfeeding }(\sim 0.002 \text {, range } 0.0016-0.01) \text {, the number of follow-up PCRs per } 100 \\
\text { HIV-exposed breastfed infants saved by doing a screening HRT at } 9 \text { months compared with } \\
\text { post-weaning PCR testing, depending on low, medium and high probability of HIV infection } \\
\text { and low, medium and high rates of seroreversion, is shown. }\end{array}$} \\
\hline
\end{tabular}

weaning PCR testing with 9-month HRT testing reduces the number of follow-up PCR tests done in all scenarios by $>50 \%$. The differences ranged from $51 \%$ to $59 \%$ with a low seroreversion rate, from $71 \%$ to $79 \%$ if the seroreversion rate was middle range, from $81 \%$ to $89 \%$ with a high seroreversion rate, from $59 \%$ to $89 \%$ with a low monthly probability of infection, from $58 \%$ to $88 \%$ with a middle estimate of infection rates, and from $51 \%$ to $81 \%$ with a high probability of infection (Table 1). The number of HIV PCRs done under the proposed 9-month HRT testing did not change by a large amount with different values for the probability of infection per month of breastfeeding, with no more than eight HIV PCR tests saved between the low and high estimates, because the main burden of HIV PCR tests carried out was for infants who have not seroreverted and will need an HIV PCR test to confirm or exclude HIV infection. Consequently, the number of HIV PCR tests done was very sensitive to the seroreversion rates used, with 30 HIV PCR tests saved between the low and high seroreversion estimates (Table 1).

\section{Discussion}

In this hypothetical calculation that sought to evaluate the potential impact of integrating HRT into the 9-month routine EPI visit, we show that the number of HIV PCR tests can be reduced by $>50 \%$ in all scenarios. This would reduce the costs associated with HIV PCR testing and the need for follow-up visits in children testing negative with HRT and no longer breastfeeding. Additionally, infants infected as a result of breastfeeding, masked intrapartum infection or late maternal infection, provided that women are tested 3-monthly post partum as recommended in the guidelines, will be diagnosed earlier than with the current $\mathrm{NDoH}$ guidelines. Currently infants infected with HIV postnatally who are breastfeeding at 9 months would only be tested 6 weeks after cessation of breastfeeding, if symptomatic or at 18 months, resulting in late diagnosis and late cART initiation. Considering the low rates of post-cessation and 18-month HIV testing, it is likely that these children would continue for many years without being diagnosed, eventually presenting ill and immunocompromised in late childhood or early adolescence. ${ }^{[14]}$ Routine testing at 9 months, integrated into the EPI, is more likely to be implemented than post-breastfeeding cessation testing that may not coincide with EPI visits. It would also allow for collection of routine data for monitoring the postnatal transmission rate at 9 months of age in a breastfeeding population, data that are not available programmatically at present.

Implementation of a 9-month HIV testing strategy requires consideration of potential barriers. The strategy requires buy-in from
EPI nurses, who may be unable or reluctant to include HIV testing in the 9-month EPI visit because of the added workload. SA's routine health data sources in 2012/13 demonstrate 94\% immunisation coverage in the first year of life and $77 \%$ at 18 months of age, whereas the infant HIV testing rate at 6 weeks was $73 \%$ with no routine data available for 18-month HIV testing. ${ }^{[2,3]}$ Attendance at routine EPI visits therefore does not automatically translate into HIV testing at these visits. The emphasis placed on 6-week HIV PCR testing dramatically increased coverage from $33 \%$ in 2008 to $74 \%$ in $2012,{ }^{[2]}$ during which time 18-month HIV testing stagnated. Prioritising HIV testing in the maternal and child health programme would be required to improve HIV testing rates at 9 and 18 months of age.

The SA HCT policy recommends provider-initiated counselling and testing for children at every contact with a healthcare facility. Since breastfed HEU children are at daily risk of HIV transmission, there is no harm in using HRT more frequently in infants aged $\geq 9$ months. HIV testing in a $\geq 9$-month-old child, identified as being 6 weeks post weaning, is indicated to determine final HIV status following maternal HIV exposure. Healthcare workers will also require education regarding the different interpretation of the HRT results in children $<18$ months of age, indicating HIV exposure if positive and not necessarily HIV infection. The consequences of failing to convey this message will result in HEU infants who serorevert later than 9 months of age being labelled as HIV-infected and initiated on lifelong therapy.

There are barriers to HIV testing in children that are not encountered in adults. A child may be accompanied to EPI visits by a caregiver who is not the parent, legal guardian or primary caregiver and is unable to provide consent for HIV testing on behalf of the child. In a study from Tanzania, children were five times more likely to be tested if they were brought to the clinic by a biological HIVinfected parent. ${ }^{[15]}$ If caregivers want to avoid testing themselves and/or their infants, there may be an adverse effect of decreased attendance at the 9-month immunisation visit. However, these barriers have not been reported in the current PMTCT programme at the 6-week visit. The nationally representative SA PMTCT evaluation study demonstrated that $97 \%$ of infants attending the 6-week EPI visit were accompanied by their mothers and caregivers, and there was high (94\%) acceptance and willingness to undergo HIV testing for themselves and their infants. ${ }^{[4]}$ Ongoing monitoring for barriers to the 9-month HIV testing strategy will be necessary.

As with any diagnostic test, false-negative results can occur, but the risk of failing to identify an HIV-infected infant can be minimised by an appropriately validated HRT and conducting concurrent clinical examinations. ${ }^{[11]}$ In programme settings, false negatives may occur because of incorrect use of the test, such as not waiting the recommended time for the true result on HRTs with 15 minutes or longer turnaround time. This can be minimised by using an HRT with a short turnaround time, which may also increase acceptability of the test to staff and clients accessing testing. Sherman et al. ${ }^{[11]}$ have shown that Insti HRT is a highly specific test for exclusion of HIV infection in this setting, has a 1-minute turnaround time for ease of use in a busy EPI clinic, and reduces the time barrier to implementing this new intervention in an already overwhelmed system.

\section{Study limitations}

This model has limitations. Owing to a lack of local data we assumed that women would breastfeed for 12 months as recommended by guidelines, but this may be an over- or underestimate, as women breastfeed for variable time periods. Maternal refusal of HIV testing for themselves and their infants was not factored into the model. Infants infected as a result of late maternal seroreversion or masked 
intrapartum infections were not included, but given that these would be the same in either testing strategy, the reported outcomes are unlikely to be affected. We did not model a detailed costing of 9-month HRT implementation, particularly the cost of training healthcare workers, and are only able to make assumptions based on the reduction in the number of HIV PCRs required.

\section{Conclusions}

If SA were to adopt the WHO recommendation to test all HEU infants at the 9-month EPI visit with an HRT and a confirmatory HIV PCR for those testing positive, more HIV-infected infants would be identified earlier, with the potential benefit of early cART initiation and monitoring of postnatal transmission rates at 9 months of age. This model suggests that replacing post-weaning testing with 9-month HIV testing would save costs for the country in terms of HIV tests performed. Training healthcare workers to interpret positive HRT results in children $<18$ months of age is likely to increase costs initially. Ongoing efforts would be required to improve implementation of testing at 9 and 18 months to determine the extent of and properly manage the paediatric HIV epidemic. A multicentre, nationwide pilot study would validate these findings and allow for a formal cost analysis of the introduction of a 9-month immunisation HRT.

\section{References}

1. UNAIDS. 2013 progress report on the global plan towards the elimination of new HIV infections among children by 2015 and keeping their mothers alive. http://www.unaids.org/sites/default/files/ media_asset/20130625_progress_global_plan_en_0.pdf 2013 (accessed 3 March 2015).
2. Sherman G, Lilian R, Bhardwaj S, Candy S, Barron P. Laboratory information system data demonstrate successful implementation of the prevention of mother-to-child transmission programme in South Africa. S Afr Med J 2014;104(3):235-238. [http://dx.doi.org/10.7196/SAMJ.7598]

3. Massyn N, Day C, Dombo M, Barron P, English R, Padarath A, and editors. District Health Barometer 2012/13. Durban: Health Systems Trust, 2013.

4. Goga A, Dinh T, Jackson D, for the SAPMTCTE study group. Evaluation of the Effectiveness of the National Prevention of Mother-to-Child Transmission (PMTCT) Programme Measured at Six Week Postpartum in South Africa, 2010. South African Medical Research Council, National Departmen of Health of South Africa and PEPFAR/US Centers for Disease Control and Prevention. 2012. http:// www.mrc.ac.za/healthsystems/SAPMTCTE2010.pdf (accessed 15 January 2015).

5. South African National Department of Health. Clinical Guidelines: PMTCT (Prevention of Motherto-Child Transmission). http://www.sahivsoc.org/upload/documents/NDOH_PMTCT.pdf (accessed 10 November 2014)

6. Grimwood A, Fatti G, Mothibi E, Eley B, Jackson D. Progress of preventing mother-to-child transmission of HIV at primary healthcare facilities and district hospitals in three South Africa provinces. S Afr Med J 2012;102(2):81-83.

7. Drake AL, Wagner A, Richardson B, John-Stewart G. Incident HIV during pregnancy and postpartum and risk of mother-to-child HIV transmission: A systematic review and meta-analysis. PLoS Med 2014,11(2):e1001608 [http://dx.doi.org/10.1371/journal.pmed.1001608]

8. Rollins N, Mahy M, Becquet R, Kuhn L, Creek T, Mofenson L. Estimates of peripartum and postnatal Rollins N, Mahy M, Becquet R, Kuhn L, Creek T, Mofenson L. Estimates of peripartum and postnatal
mother-to-child transmission probabilities of HIV for use in Spectrum and other population based models. Sex Transm Infect 2012;88(suppl 2):i44-i51. [http://dx.doi.org/10.1136/sextrans-2012-050709] 9. Nielsen-Saines K, Watts $\mathrm{H}$, Veloso VG, et al. Three postpartum antiretroviral regimens to prevent intrapartum HIV infection. N Engl J Med 2012;366(25):2368-2379. [http://dx.doi.org/10.1056/NEJMoa1 108275]

10. World Health Organization. WHO Recommendations on the Diagnosis of HIV Infection in Infants an Children. 2010. http://whqlibdoc.who.int/publications/2010/9789241599085_eng.pdf(accessed 11 July 2012).

11. Sherman GG, Lilian RR, Coovadia AH. The performance of 5 rapid human immunodeficiency virus tests using whole blood in infants and children: Selecting a test to achieve the clinical objective. Pediatr Infect Dis J 2012;31(3):267-272. [http://dx.doi.org/10.1097/INF.0b013e31823752a0]

12. Vergueta S, Jassat W, Hedberg C, Tollman S, Jamison DT, Hofman KJ. Measles control in sub-Saharan Africa: South Africa as a case study. Vaccine 2012:30(9):1594-1600. [http://dx.doi.org/10.1016/]. vaccine.2011.12.123]

13. Chasela CS, Hudgens MG Jamieson DI et al. Maternal or infant antiretroviral drugs to reduce HIV1 transmission. N Engl J Med 2010,362(24):2271-2281. [http://dx.doi.org/10.1056/NEJMoa0911486] Ferrand RA, Munaiwa L, Matsekete J, et al. Undiagnosed HIV infection among adolescents seeking primary health care in Zimbabwe. Clin Infect Dis 2010;51(7):844-851. [http://dx.doi.org/10.1093/cid/cis271]
he

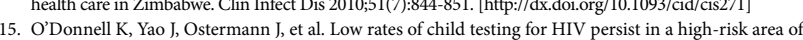
15. O'Donnell K, Yao J, Ostermann J, et al. Low rates of child testing for HIV persist in a high-risk area of
East Africa. AIDS Care: Psychological and Socio-medical Aspects of AIDS/HIV 2014;26(3):326-331. [http://dx.doi.org/10.1080/09540121.2013.819405]

Accepted 1 July 2015 